\title{
A Study on Biological and Catalytic Activity of CSB Derivative and its Cobalt and Nickel Complexes
}

\author{
R. SURESH ${ }^{1}$, M. DEEPA ${ }^{2 *}$, P. MOGANAVALLY ${ }^{3}$ and P.N. SUDHA ${ }^{4}$ \\ ${ }^{1}$ Research and Development Centre, Bharathiyar University, Coimbatore, India; \\ ${ }^{2}$ Department of Chemistry, Muthurangam Govt. Arts College, Vellore, India; \\ ${ }^{3}$ Department of Chemistry, Shanmuga Industries Arts College, Thiruvannamalai, India; \\ ${ }^{4} P G$ \& Research Department of Chemistry, D.K.M. College for Women, Vellore, India; \\ ${ }^{*}$ Corresponding author E-mail: deeparam79@gmail.com \\ http://dx.doi.org/10.13005/ojc/320521
}

(Received: August 21, 2016; Accepted: October 03, 2016)

\begin{abstract}
The Carboxymethyl chitosan Schiff base (CSB) derivative and its cobalt and nickel complexes (CSB-Co \& CSB-Ni) have synthesized. The FTIR results showed the interaction with the amine of Carboxymethyl chitosan and aldehyde confirming the Schiff base formation. The XRD results, in comparison to CMC, displayed weaker peaks, which shows a decrease in crystalline properties and also exhibits the amorphous nature. The catalytic activity of the prepared CSB and its metal complexes were in the order, CSB > CSB-Co > CMC > CSB-Ni complex. From the DPPH studies, ALP assay and cytotoxic studies, the result proves that the prepared sample is non-toxic, biocompatible and has good antioxidant activity.
\end{abstract}

Keywords: Carboxymethyl chitosan; Schiff base; biological activity; catalytic activity.

\section{INTRODUCTION}

Biopolymers were found in nature and can be easily extracted. Hence, the present work deals with polysaccharides, such as chitosan, an amino polysaccharide ${ }^{1}$. The properties and applications of chitosan can be enhanced through modification by physical or chemical processes ${ }^{2}$. Carboxymethyl chitosan (CMC), which is biocompatible and biodegradable, is formed when the mono- chloroacteic acid reacted with the chitosan in alkaline condition ${ }^{3}$.

Schiff bases have recently assumed much attention because they have been found to be biologically active and have found uses in biology, medicine as well as in industry. The properties of formation of polynuclear complexes ${ }^{4}$ depend on the nature of the metal ion as well as on the nature of the ligands. Hence, in the present 
study, CSB (Carboxymethyl chitosan Schiff base) derivative was prepared using Carboxymethyl chitosan with 3, 4-dimethoxy Benzaldehyde since they offer opportunities to induce biological activity. Furthermore, metal with Schiff base play a vital role in biological systems. Hence, metals like cobalt and nickel were incorporated with the CSB derivative and their characterization and biological activity were studied. CSB derivative and its complexes were characterized by Fourier Transform Infrared Spectra (FTIR), XRD, DSC, TGA, and SEM studies. Furthermore, biological activity, which includes antioxidant activity, in vitro cytotoxicity, ALP study and catalytic activity, was investigated for CMC, CSB, CSB-Co and CSB-Ni complexes.

\section{Experimental Section}

\section{Materials}

CMC and 3, 4-dimethoxy Benzaldehyde, were purchased from India sea foods, Cochin and Sigma Aldrich, India. Cobaltous chloride, nickel chloride used was of analytical grade.

\section{Preparation of CSB Complex Synthesis of CSB}

$A$ required amount of $\mathrm{CMC}$ along with ethanol and water were stirred at room temperature for 30 minutes. To this add 3, 4-dimethoxy Benzaldehyde and heated to $60^{\circ} \mathrm{C}$ for 12 hours under water bath. At the end, the product was washed with ethanol and dried vacuum for 24 hours 5 .

\section{Synthesis of CSB Metal complexes}

$0.5 \mathrm{mmol}$ of CSB with ethanol is magnetically stirred for 5 hours and to this add $0.5 \mathrm{mmol}$ ethanolic solution of $\mathrm{CoSO}_{4}$, continue stirring for 15 hours. The crude product (CSB-Co) obtained was washed with ether and dried in a vacuum. The same procedure was followed to prepare CSB nickel (II) (CSB-Ni) complex. The structure of the prepared CSB metal complexes was shown in Figure 1.

\section{Characterization of the Samples}

FTIR of CSB and its metal complexes were recorded at $400-4000 \mathrm{~cm}^{-1}$ frequency using Thermo Nicolet AVATAR 330 spectrophotometer. By using an XRD - SHIMADZU XD - D1 with Ni filter and $\mathrm{Cu}$ Ká radiation source, the relative intensities were measured $10^{\circ}$ to $90^{\circ}$. To verify the crystalline properties the SEM (scanning electron microscope) has taken by cutting the samples into small pieces and wiped with Au-Pd layer by a sputter coat unit (VG Microtech, Uckfield, UK) and the crosssection topography was analyzed with Cambridge Stereoscan 440 Scanning Electron Microscope (Leica, Cambridge UK).

\section{Evaluation of Biological Activity In vitro cytotoxicity and cell proliferation assay \\ Cell viability on the CSB and its metal} complexes was measured using MTT assay, which is based on bio-reduction of enzymes in living active cells. A fresh culture medium is prepared using 400 $\mu \mathrm{L}$ of MTT at $37^{\circ} \mathrm{C}$ for 4 hours in absence of light. By removing the unreacted one, add DMSO (400 $\mu \mathrm{L}$ ) was added to dissolve purple formazan and the absorbance were measured using spectrophotometer at $540 \mathrm{~nm}$ with a GENios $\AA$ microplate reader (Tecan Austria $\mathrm{GmbH}$, Austria). On comparing with the control, the results were produced.

\section{Alkaline phosphatase assay}

After incubation, the cells along with PBS buffer $(25 \mathrm{mM}$ carbonate buffer with $0.1 \%$ Triton X-100) are incubated for half an hour. To the pretreated cells add $1.5 \mathrm{mM} \mathrm{MgCl}$ and $15 \mathrm{mM}$ para-nitro phenyl phosphate ( $\mathrm{p}-\mathrm{NPP})$ and again incubated. Now the p-NPP was converted as $p$-Nitro phenol and inorganic phosphate. The ALP assay was found from the absorbance measured at $405 \mathrm{~nm}$ spectrophotometer (Tecan Austria GmbH, Austria).

\section{Antioxidant activity}

The scavenging activity was determined using the different method. An ethanolic solution $(0.1 \mathrm{~mL})$ of $D P P H(0.1 \mathrm{mmol} / \mathrm{L})$ was incubated $20 \mathrm{~min}$ at $30^{\circ} \mathrm{C}$ for with different concentrations of the samples $(0.1 \mathrm{~mL})$ and the absorbance were measured at $517 \mathrm{~nm}$ against a blank.

$$
\text { Scavenging activity }(\%)=\left[1-\mathrm{A}_{\text {sample }} / \mathrm{A}_{\text {control }}\right] \times 100 \%
$$

\section{Catalytic activity}

$0.05 \mathrm{~g}$ of the samples in acetonitrile $(10 \mathrm{ml})$ with $10 \mathrm{mmol}$ of $\mathrm{H}_{2} \mathrm{O}_{2}$ solution is magnetically stirred continuously fitted with a water condenser under aerobic conditions. To this add $5 \mathrm{mmol}$ of cyclohexane under $70^{\circ} \mathrm{C}$ for an half a day. Separate the Aliquots and collect it for the $8^{\text {th }}$ hour and the 
$12^{\text {th }}$ hour. Blank experiments were conducted for the sample without $\mathrm{H}_{2} \mathrm{O}_{2}$ and the other sample without the metal complex. All the samples were measured with a chromatogram (Hewlett-Packard gas chromatogram -HP 6890) having FID detector.

Conversion \% of Cyclohexane $=100 \times[$ Initial \%-

Final\%] /Initial\%

\section{RESULTS AND DISCUSSION}

\section{FTIR Studies}

The FTIR of Carboxymethyl chitosan shown in Figure-2 has taken for comparison only. On comparing, the broad peak appeared at 3420 $\mathrm{cm}^{-1}$ corresponds to the intra-molecular hydrogen bonding is at high intensity for Carboxymethyl
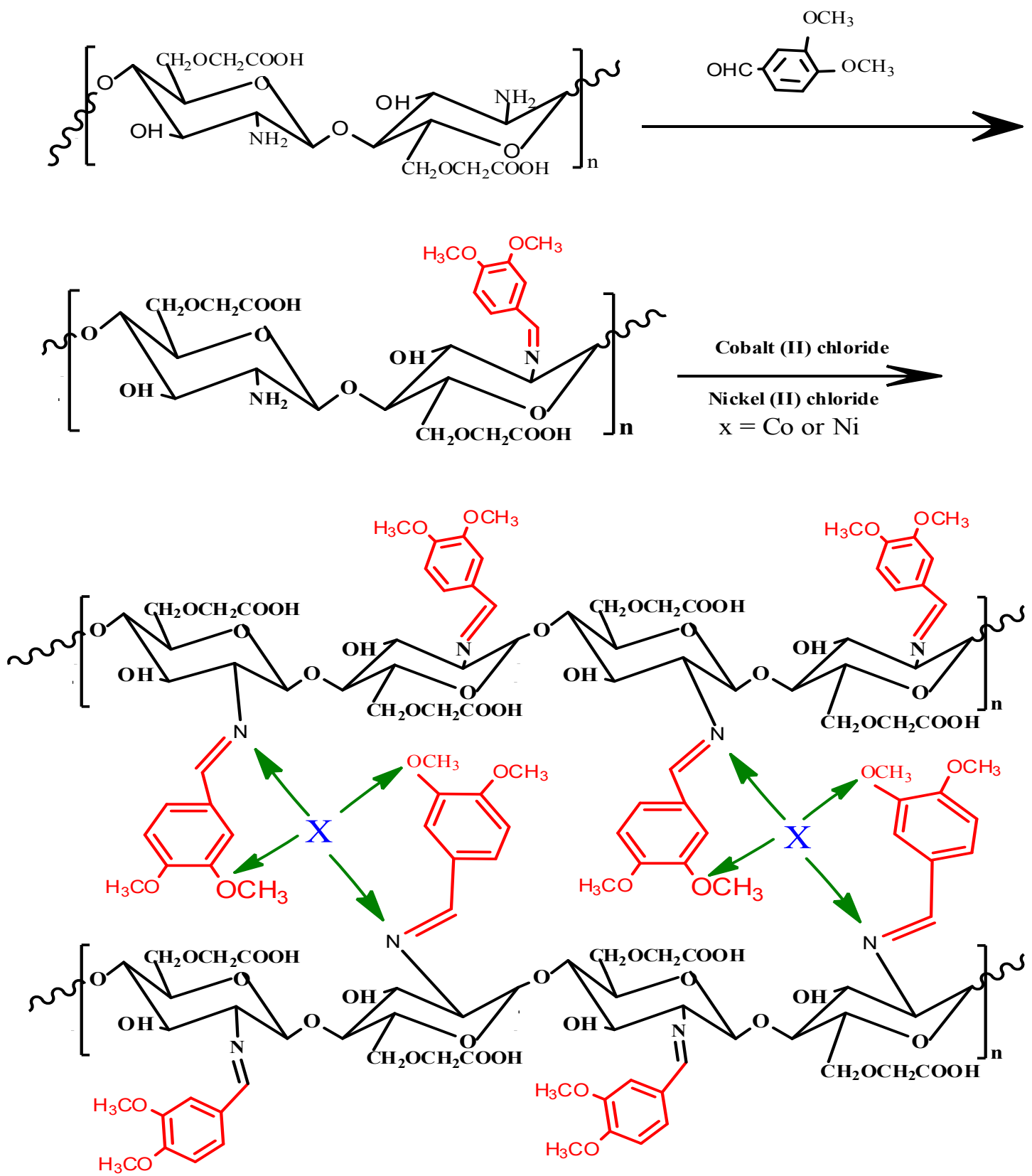

Fig. 1: Structure of CSB metal complex 
chitosan and the intensity decreases for CSB and its metal complexes. This peak has shifted to 3393 $\mathrm{cm}^{-1}$, due to the aldehyde and polymer involved in hydrogen bonding. The stretching of $\mathrm{C}-\mathrm{H}$ bonds, $\mathrm{C}=\mathrm{O}$ stretching and $\mathrm{NH}$ bending appeared at 2918, 1725 and $1630 \mathrm{~cm}^{-16}$. A peak at $1430 \mathrm{~cm}^{-1}$ is due to the $\mathrm{C}-\mathrm{N}$ axial stretching and $\mathrm{N}-\mathrm{H}$ angular deformations in CSB derivative and in metal complexes appeared at $1383 \mathrm{~cm}^{-1}$. In CSB derivative the peak appeared at $1644 \mathrm{~cm}^{-1}$ confirms the Schiff base formation. The band around $500-600 \mathrm{~cm}^{-1}$ indicates the $\mathrm{M}-\mathrm{N}$ and $\mathrm{M}-\mathrm{O}$ stretching confirming the metal present in the prepared CSB metal complexes.

\section{XRD Studies}

To determine the crystalline properties and structure X-ray diffraction analysis is used ${ }^{7}$. The X-ray pattern of the CSB derivative and its cobalt and nickel complexes were shown in Figure 3.On comparison with $\mathrm{CMC}$ alone, the CSB exhibits broader peak with low intensity at $2 \mathrm{è}=20^{\circ}, 28^{\circ}$ and $46^{\circ}$. The decrease in crystallinity and increase in the amorphous nature
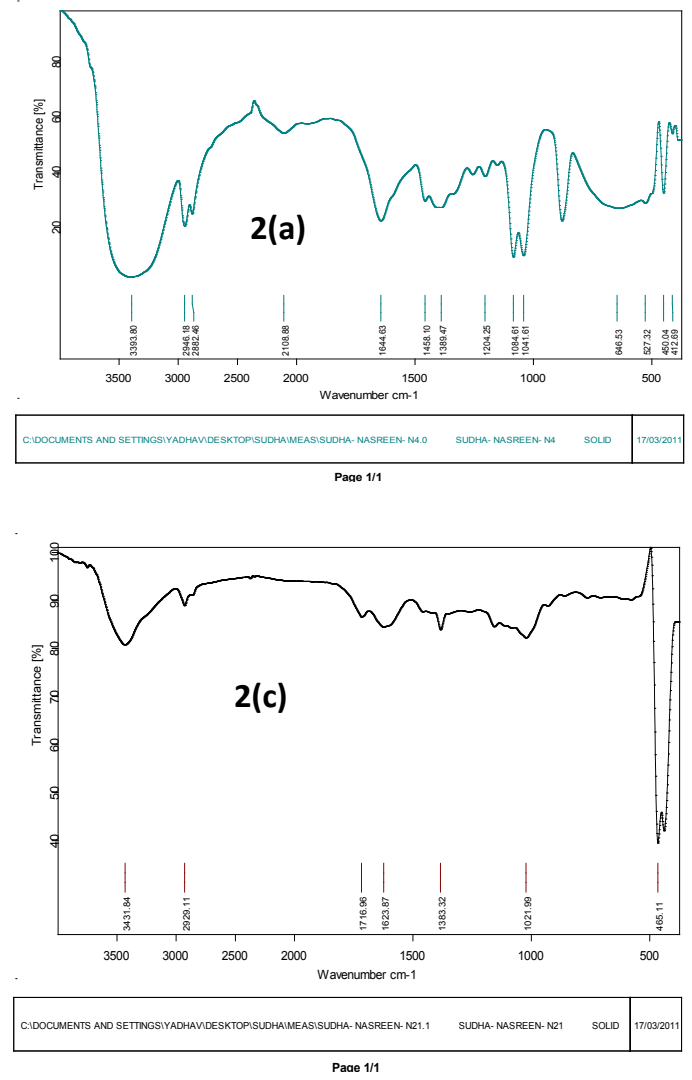

Fig. 2: FT-IR images of (a) CMC (b) CSB (c) CSB-Co \& (d) CSB-Ni complex

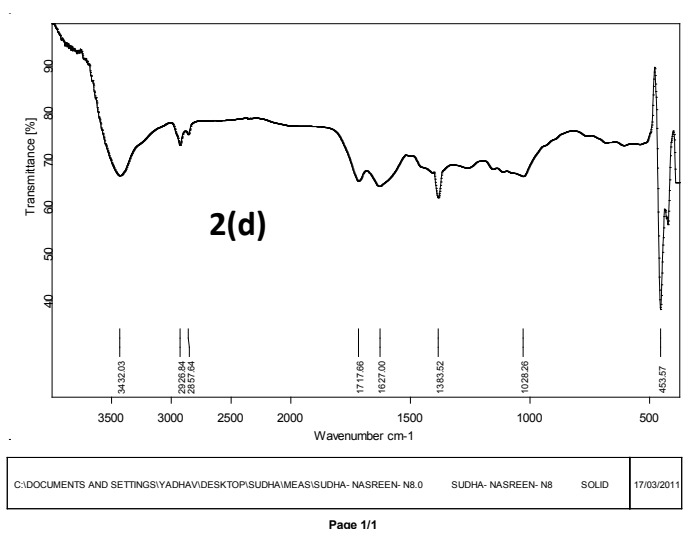

confirms the formation of Schiff base complexes. In the cobalt complex, only one broad peak appears at around $20^{\circ}$ and all the other peaks disappear. In the Schiff base nickel complex, the broad peak appears around $20^{\circ}$ and $42^{\circ}$. These results confirm the coordination of metal with Schiff base complex.

\section{SEM Analysis}

The morphologies of CSB complexes were analyzed and shown in Figure 4. The surface of CMC is smooth and uniform. With the addition of aromatic aldehyde, the surface of the formed CMC Schiff base displayed rough and dense surfaces, suitable for biomedical applications. On further addition of metals like cobalt and nickel, the Schiff base metal complex exhibits irregular morphology. Due to the metal-ligand coordination with Cobalt CSB complex, results in a flat smooth surface. However, the CSB-Ni complex exhibits a rough surface with a greater number of pores suitable for adsorption and biomedical applications.

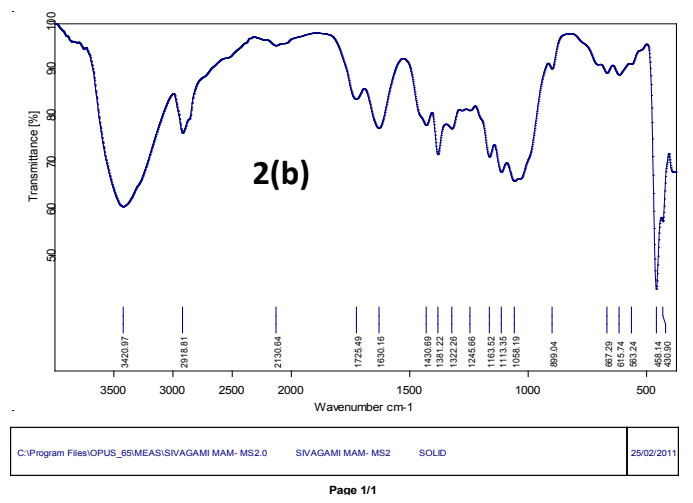




\section{In Vitro Cytotoxicity}

The viability assays play a vital role in toxicity studies that determine the cellular response to a toxicant. Generally, cell viability gives details about the cell death and its metabolic activities ${ }^{8}$. The cytotoxic effect of CMC, CSB and its cobalt and nickel complex was evaluated in vitro against human breast carcinoma (MCF-7) cell lines by MTT assay and
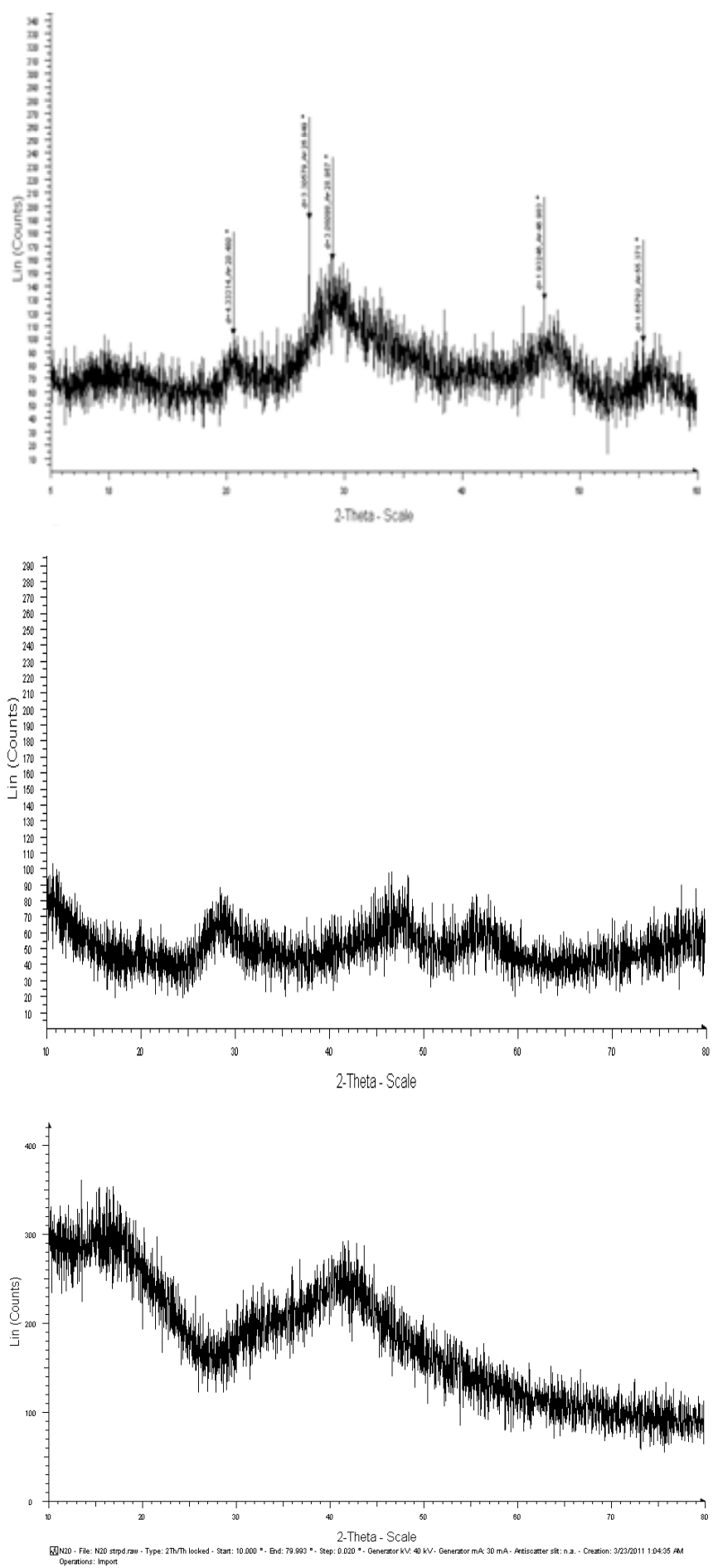


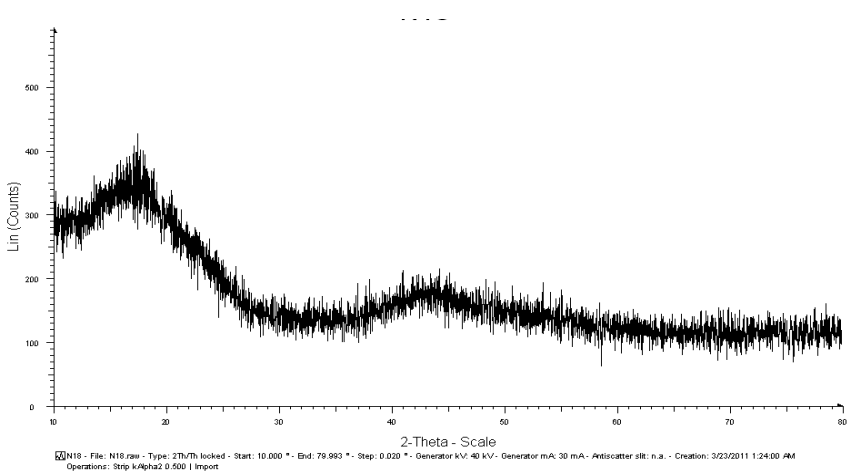

Fig. 3: XRD pattern of (a) CMC (b) CSB (c) CSB-Ni \& (d) CSB-Co complex
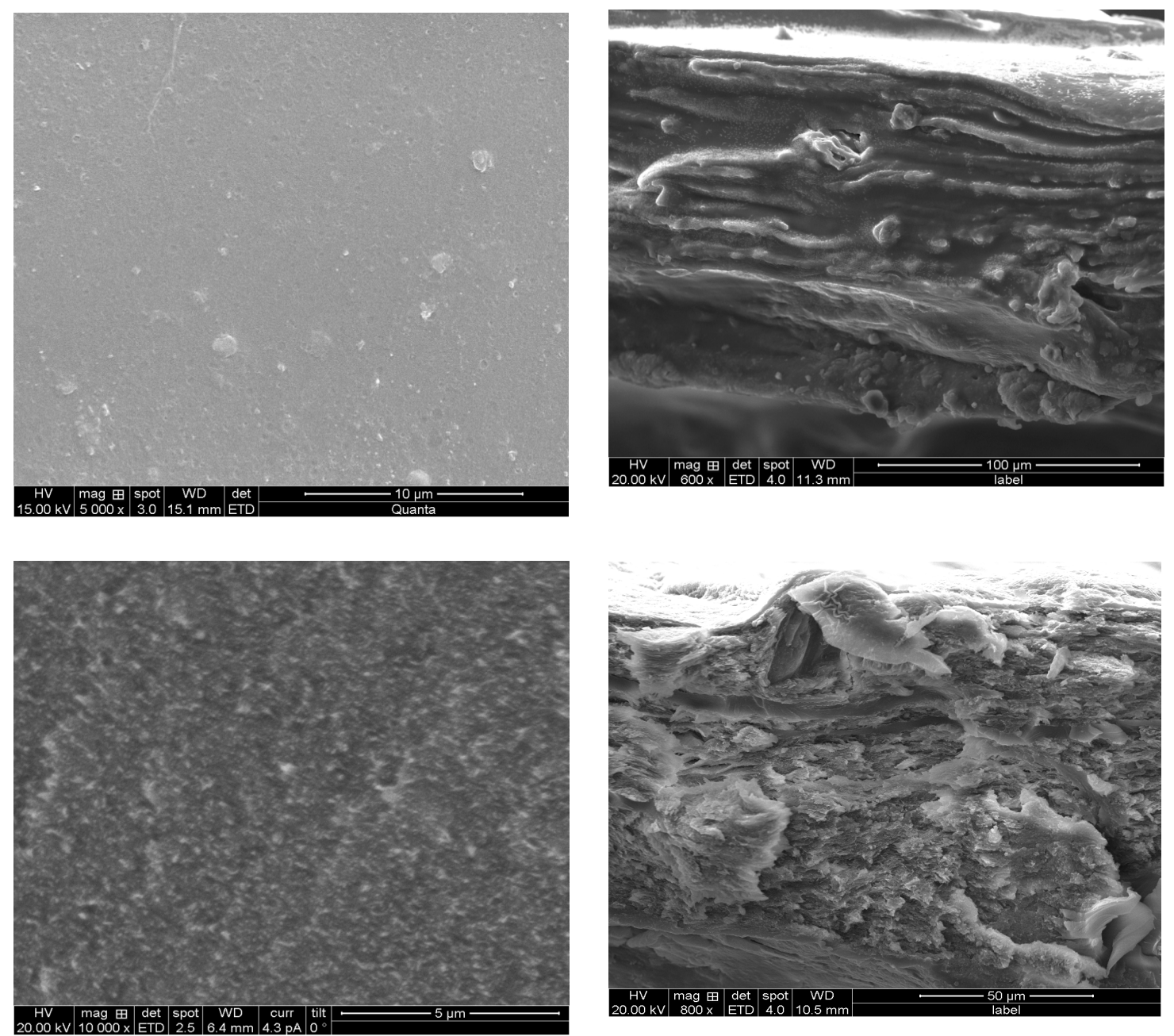

Fig. 4: SEM images of (a) CMC (b) CSB (c) CSB-Co \& (d) CSB-Ni complex 
the results shown in the Figure 5. The percentage viability of MCF-7 cell lines increased as the number of days increased from 1 to 14 . On comparing with the Cobalt CSB complex, CSB-Ni complex exhibits higher percentage of cell viability. The percentage cell viability as a function of time on the prepared sample shows the cellular compatibility and may be useful for tissue engineering applications ${ }^{9}$. Thus, the results proved that the prepared samples are good material, non-toxic, and compatible, which can be further used in various biomedical applications.

\section{Alkaline Phosphatase (ALP) Assay}

The Phosphorylated CSB cobalt and nickel complex were analysed using ALP test on the MCF-7 cell line and reported in Figure 6. From the results, we concludethe ALP activity was found to increase from day 1 to day 14 . On comparing the CSB, CSB-Co,

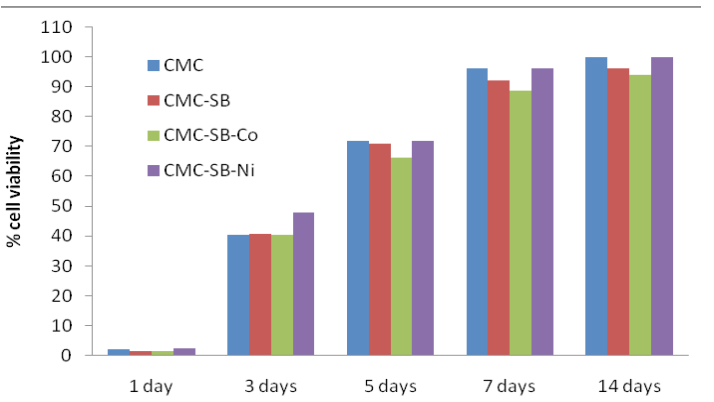

Fig. 5: Cell proliferation assay

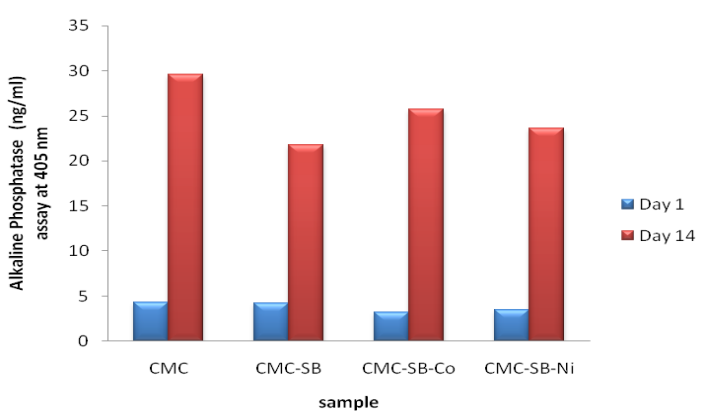

Fig. 6: ALP activity

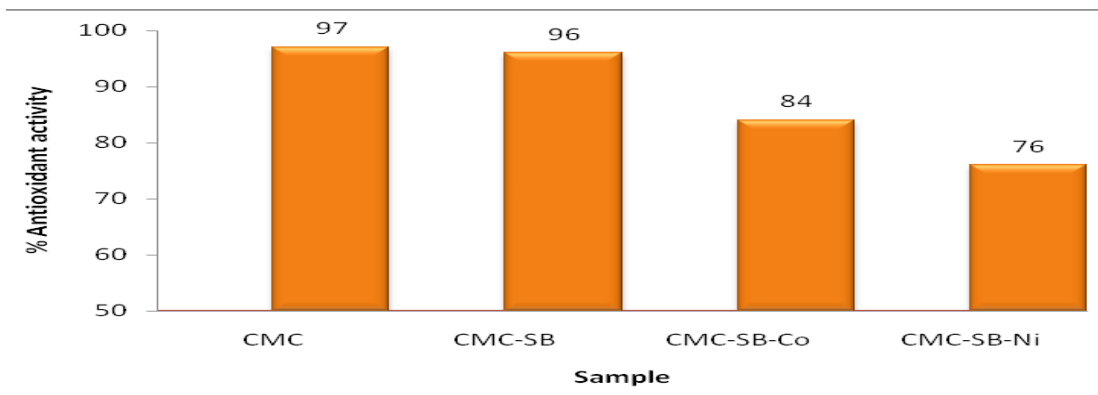

Fig. 7: Antioxidant activity

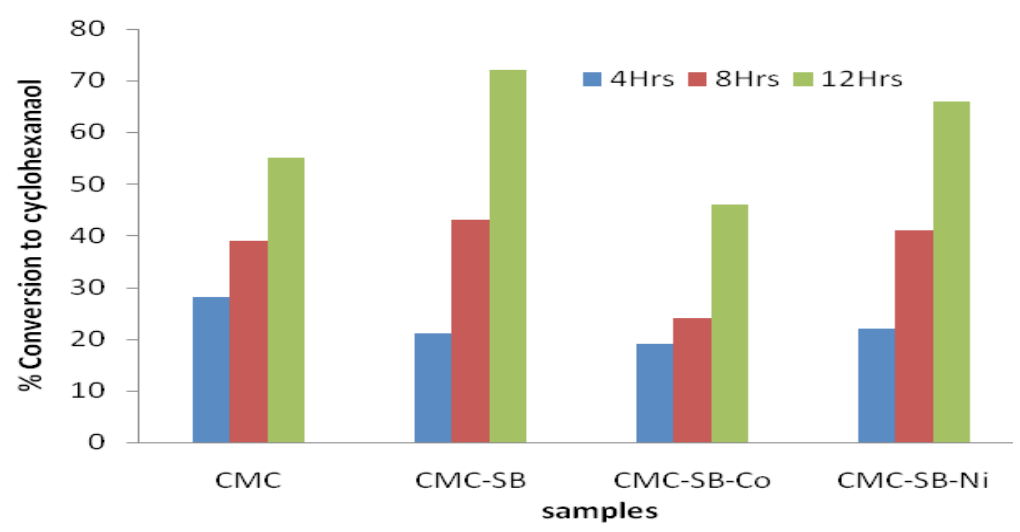

Fig. 8: Catalytic oxidation 
and CSB-Ni, it was found that the ALP level regularly increased in the presence of cobalt complexes significantly as compared to the other samples.

The ALP activity of prepared samples was ranked as follows:

$\mathrm{CMC}>\mathrm{CSB}-\mathrm{Co}>\mathrm{CSB}-\mathrm{Ni}>\mathrm{CSB}$

\section{Antioxidant Activity}

The antioxidant potential of CSB complexes was studied and the change in optical density of DPPH radicals was monitored and given in Figure 7.

The scavenging activity for CSB is $96 \%$, which is nearly equal to the activity for Carboxymethyl chitosan. However, in the presence of metal Schiff base cobalt and nickel complex, the scavenging activity goes on decreasing. On comparing cobalt and nickel Schiff base complex, cobalt Schiff base complex exhibits higher activity than nickel Schiff base complex.

\section{Catalytic Activity}

The selective oxidation of cyclohexane to cyclohexanol is of great industrial importance ${ }^{10}$. Figure 8 represent the catalytic activity of $\mathrm{CMC}$,
CSB, CSB-Co, and CSB-Ni complexes. The results confirmed that the catalytic activity was more pronounced by CMC Schiff base complex, $72 \%$ after 12 hours. On increasing the time, the conversion percentage also increased up to 12 hours for all the derivatives and drastic changes noted from 8 to 12 hours.

\section{CONCLUSION}

The synthesized CSB metal complexes were characterized by FTIR, XRD and SEM studies. A broader peak in FTIR study confirmed the formation of CSB and its cobalt and nickel complexes. The cell viability graph indicated of cellular compatibility and further use in tissue engineering applications. The results suggest that the prepared CSB and its cobalt and nickel complexes are good material, non-toxic and compatible. There is an increase in the ALP activity on CSB-Ni complex compared to the other samples. The antioxidant activity was more pronounced in the Schiff base however, this decreased after the metal Schiff base was formed. Catalytic oxidation is more pronounced in the CSB compared to the others. The evaluation of biological activity confirms that the prepared samples can be used for various biomedical applications.

\section{REFERENCES}

1. Kumar, M. Reactive and Functional Polymers, 2000, 46, 1-27.

2. Moganavally, P.; Deepa, M, Sudha, M.; Suresh, R.; Orient. J. Chem., 2016, 32, 441-453.

3. Chen, X.G.; Wang, Z.; Liu, W.S.; Park, H.J. [J] Biomaterials 2002, 23, 4609-4614.

4. De Geest, D.J.;Noble, A.; Moubaraki, B.; Murray, K.S.; Larsen, D.S.; Brooker, S. Dalton Tr. 2007, 28, 467-475.

5. Guinesi, L.S.; Cavalheiro, E.T.G. Thermochim Act, 2006, 449, 1-7.

6. Chen, X.G.; Park, H.J. Carbohydr. Polym. 2003, 53, 355.
7. Sameer Pradhan; Wayne Ward; Kadri Hacioglu; James Martin; Dan Jurafsky. Semantic role labeling using different syntactic views, In Proceedings of the Association for Computational Linguistics 43rd annual meeting (ACL- 2005), Ann Arbor, MI.

8. Willingham, M.C. J Histochem Cytochem 1999, 47, 1101-1109.

9. Han, J.; Zhou, Z.; Yin, D.; Nie, J. International journal of Biological Macromolecules 2010, 46, 199-205.

10. Schuchardt, U.; Carvalho, W.A.; Spinace, E.V. Synlett 1993, 10, 713-718. 